\title{
Spectral Passband Filter With Independently Variable Center Wavelength and Bandwidth
}

\author{
Gordon Wilson, Chien-Jen Chen, Phil Gooding, and Joseph E. Ford
}

\begin{abstract}
We introduce a new class of compact grating-based spectral filters and demonstrate a two-port spectral filter that uses a single two-axis microelectromechanical systems tilt mirror to continuously tune the center wavelength across $36 \mathrm{~nm}$ and independently tune the passband 3-dB spectral width from 28 to $210 \mathrm{GHz}$. We also demonstrate the principle of a three-port channel-drop switch.
\end{abstract}

Index Terms-Optical fiber, optical microelectromechanical systems (MEMS), reconfigurable optical add-drop multiplexers, tunable optical filters.

\section{INTRODUCTION}

D YNAMIC spectral filters using diffraction gratings and free-space optics are used for add-drop switching, spectral equalization, and spectral monitoring. Existing devices fall into two distinct categories. Single-pass tunable spectral filters, including bandpass filters and spectrometers, disperse the input signal across a fixed spectral plane spatial filter (SPSF) that transmits selected signals to an output fiber or slit-apertured photodetector [1]-[3]. The SPSF is fixed, but the filter center wavelength is tuned using a tilting mirror or grating. The second class of tunable filters, including wavelength-selective add-drop switches and spectral equalizers, use a double-pass configuration where the input is dispersed across a reconfigurable SPSF, such as an array of tilting-microelectromechanical systems (MEMS)-mirror or liquid-crystal pixels, then collected into one or more outputs by a second pass through the grating [4], [5]. Here we propose and demonstrate a third class of grating-based spectral filters, "spectrum steering" filters, that combines the double-pass geometry with a fixed SPSF. A single active element, a shared MEMS mirror, steers the dispersed spectrum in two dimensions across a fixed SPSF to select among a set of pre-programmed filter shapes and tune the center wavelength of each.

Using this architecture, we experimentally demonstrate a spectral bandpass filter with independently variable center wavelength and bandwidth. We further show how the architecture could be extended to build three-port hitless channel drop filters with feedback stabilization. Tunable bandpass filters provide colorless demultiplexing ports in broadcast-and-select architectures [6]. Making the bandwidth selectable allows a single filter to select individual channels regardless of data rate

Manuscript received December 5, 2005; revised March 3, 2006.

G. Wilson is with JDSU, Milpitas, CA 95035 USA (e-mail: gordon. wilson@jdsu.com).

C.-J. Chen was with the Electrical and Computer Engineering Department, University of California San Diego, San Diego, CA 92093 USA.

P. Gooding is with OptiMedica Corp., Santa Clara, CA 95054 USA.

J. E. Ford is with the Electrical and Computer Engineering Department, University of California San Diego, San Diego, CA 92093 USA (e-mail: jeford@ucsd.edu).

Digital Object Identifier 10.1109/LPT.2006.879539
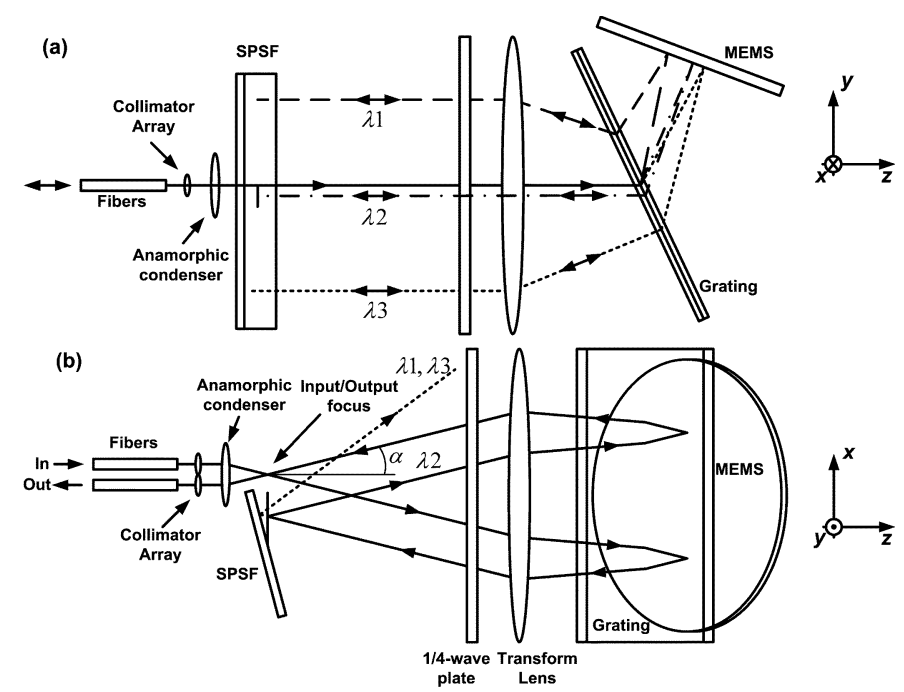

Fig. 1. Schematic of the tunable filter. (a) Top view; (b) side view. $\lambda_{2}$ is passed to the output while $\lambda_{1}$ and $\lambda_{3}$ are rejected.

or channel spacing. A blocking filter with a discontinuously adjustable passband was demonstrated using an array of MEMS mirrors in the spectral plane [7]. For applications requiring only one passband, the device presented here should be relatively inexpensive and compact due to simplified MEMS device fabrication and control electronics, the smaller field-of-view required of the imaging system, and the ability to use active alignment compensation for thermal drift.

\section{Filter DesigN}

The tunable filter geometry (Fig. 1) is a folded double-passed 4f imaging system. A single lens collimates the input signal onto a diffraction grating and focuses the dispersed signal onto the SPSF. A controlled portion of the spectrum is reflected from the SPSF back through the grating, which recombines the spectral components of the signal and directs it to the output. In Fig. 1, the SPSF passes $\lambda_{2}$ to the output but rejects $\lambda_{1}$ and $\lambda_{3}$. A single two-axis tilt mirror in the collimated-beam plane controls filter transmission by positioning the dispersed signal on the SPSF.

The optical system uses pupil-division multiplexing with anamorphic imaging to separate the input and output signal [8]. Light input to either of two standard single-mode fibers, separated by $250 \mu \mathrm{m}$, is collimated by a coaxial microlens and then focused to a common elliptical spot in the input image plane by a small anamorphic condenser lens centered on an axis midway between the two fibers, such that the chief rays of the input and output beams make an angle $\alpha$ with the axis of the condenser. The magnifications of the fiber spot size in the $x$ and $y$-directions are $M_{x}=2.25$ and $M_{x}=4.5$, respectively.

The dispersive element in this system is a Wasatch Photonics 940-lines/mm transmissive holographic grating placed between the transform lens and the MEMS tilt-mirror. The dispersed 


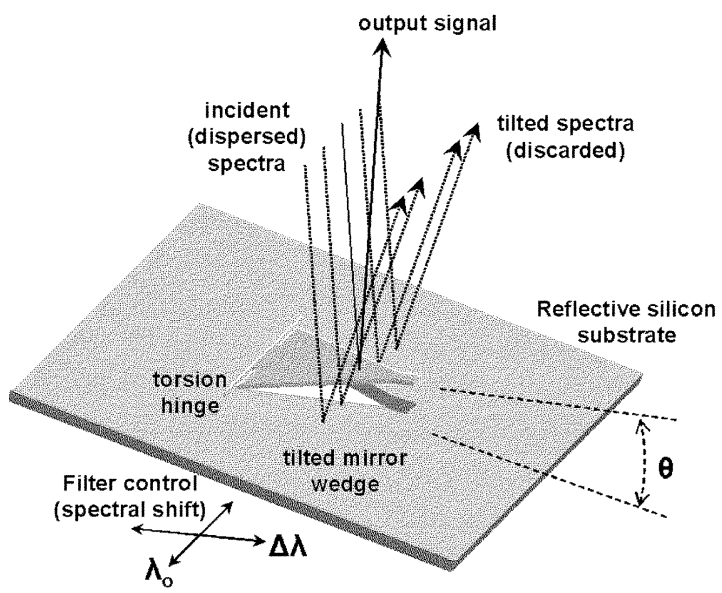

Fig. 2. Fixed SPSF, a reflective surface with a tilted wedge to direct a selectable section of the incident light to the output.

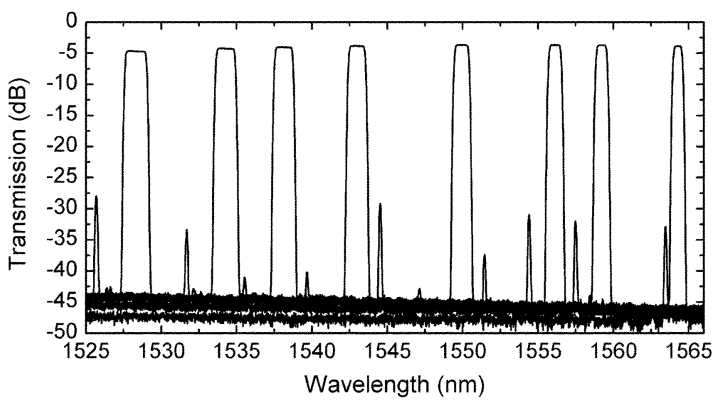

Fig. 3. Filter transmission as a function of mirror $x$-axis rotation, showing superimposed traces for eight different control voltages.

signal reflects from the mirror and is further dispersed by a second pass through the grating. The system has a quarter-wave plate between the transform lens and image plane to average the polarization dependence of the grating efficiency [4].

The MEMS mirror, a prototype beam-steering mirror from Texas Instruments, with a $4.5 \times 6-\mathrm{mm}$ aperture, is electromagnetically actuated, with a linear angular variation over $\pm 1.5^{\circ}$ in response to $\mathrm{a} \pm 2.3-\mathrm{V}$ drive and a switching speed of approximately $10 \mathrm{~ms}$. A mirror rotation of $1^{\circ}$ about the $x$-axis moves the center wavelength of the dispersed spectra by $1.1 \mathrm{~mm}$ at the SPSF, corresponding to $15 \mathrm{~nm}$ of wavelength shift.

Silicon micromachining was used to produce the static SPSF (Fig. 2), which consists of a flat reflective field and a tilted wedgeshaped reflective region. It is oriented such that any portion of the signal illuminating the tilted wedge is coupled into the output fiber, while light illuminating the surrounding region is discarded. The triangular reflective beam is hinged at its base and pushed up from below by a silicon substructure (not shown) then locked in place. In Fig. 2, the central signal illuminates the tilted portion of the SPSF and is reflected to the output. Rotating the MEMS mirror around the $x$-axis shifts the signal in the direction orthogonal to the wedge, changing the center wavelength, while rotating the MEMS mirror about the $y$-axis shifts the dispersed signal along the wedge, changing the transmission bandwidth.

\section{EXPERIMENTAL RESULTS}

The filter transmission is shown in Fig. 3 for eight different control voltages applied to the mirror $x$-axis. The passband width varies with center wavelength because the grating and
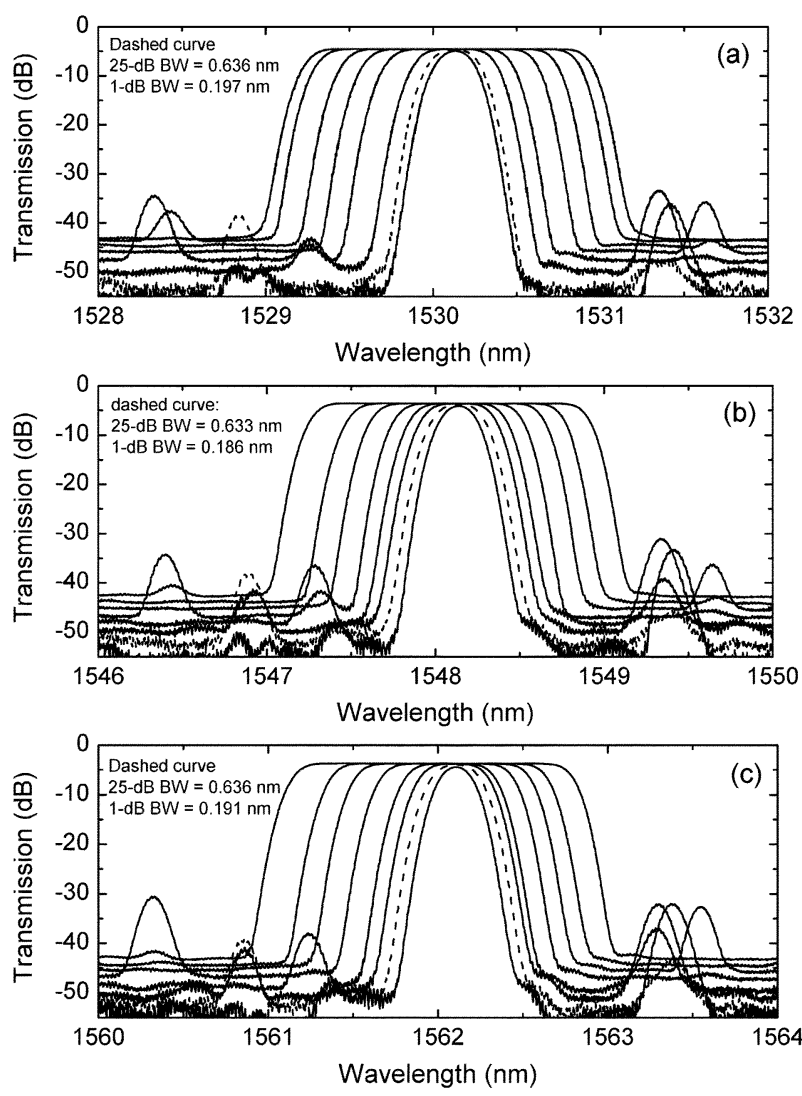

Fig. 4. Filter passband profile as a function of mirror $y$-axis rotation for three center wavelengths. The dashed curves show appropriate filter settings for demultiplexing 50-GHz spaced channels.

MEMS mirror axes are not perfectly aligned. This effect could be corrected by adjusting both mirror axis control voltages simultaneously. The total insertion loss (IL) is less than $4.6 \mathrm{~dB}$ across the full 40-nm operating spectrum. The transmission grating, which is passed four times, produces approximately $3.3 \mathrm{~dB}$ of that loss. Adjusting the azimuth of the quarter-wave plate reduced polarization-dependent loss (PDL) from a maximum of $1.7 \mathrm{~dB}$ to the operating value of $0.26 \mathrm{~dB}$. The grating limits the tuning range with increased IL at short wavelengths and PDL at long wavelengths.

The filter shape is shown for various MEMS gimbal axis voltages at three center wavelengths in Fig. 4 and exhibits low passband ripple. The nonadjacent channel crosstalk is under $-40 \mathrm{~dB}$ except for isolated sidelobes, tentatively attributed to collimating lens aberrations, $25 \mathrm{~dB}$ below the peak. The background crosstalk increases with increasing filter width because the numerical aperture of the transform lens captures more of the rejected light (shown as dotted line in Fig. 1) as the spectrum is translated along the reflective filter beam.

The resolution can be parameterized as the difference $\Delta B$ between the 1- and 25-dB full bandwidths. The number of grating lines illuminated determines the resolution. For a Gaussian input aperture we predict $\Delta B=2.82 \lambda / \pi \omega_{C} D=0.38 \mathrm{~nm}$, where $D=2.74 \times 10^{-3}$ radians $/ \mathrm{nm}$ is the double-pass grating dispersion and $\omega_{C}=\lambda f / \pi M_{x} \omega_{f}=1.33 \mathrm{~mm}$ is the collimated-beam radius. $\omega_{f}$ is the fiber mode radius, and $f=31.7 \mathrm{~mm}$ is the transform-lens focal length. The nonparallelism of the reflective beam edges (Fig. 2) of our wedge-shaped filter limits the resolution to $\Delta B=0.46 \mathrm{~nm}$ for most bandwidths [Fig. 4(a)-(c)]. 


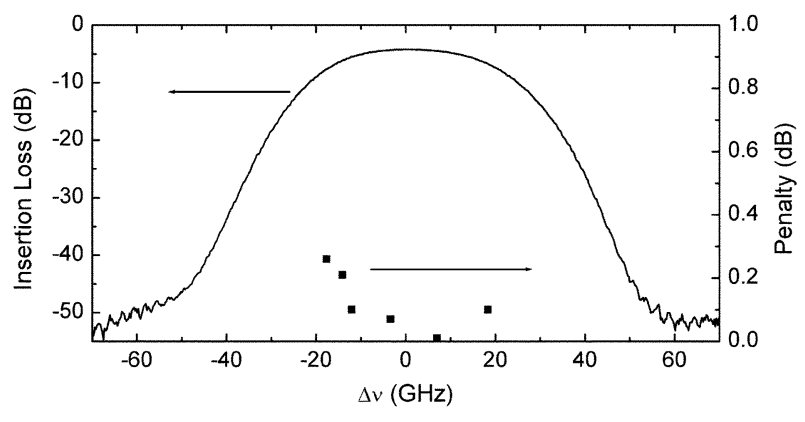

Fig. 5. IL and $10-\mathrm{Gb} / \mathrm{s}$ power penalty as a function of transmitter frequency detuning from the channel passband center.
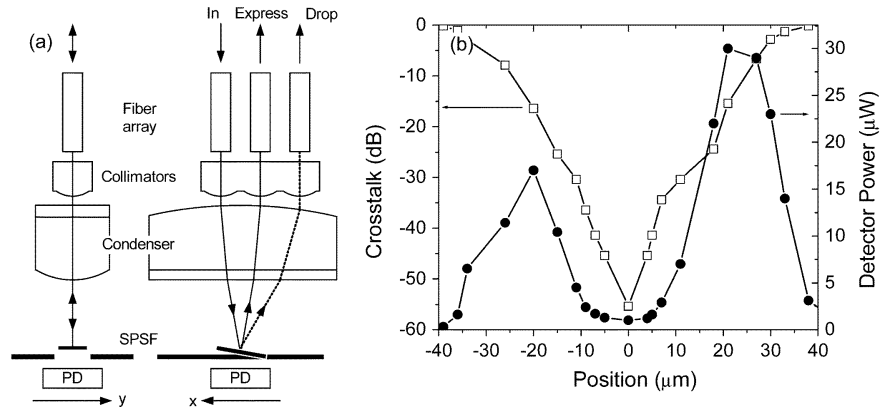

Fig. 6. (a) Three-port switch with feedback-stabilization demonstration-of-principle. PD detects light passing between gaps in the SPSF. (b) Optical power measured by PD and crosstalk from drop to express ports.

However, for the narrowest two filter settings, the spectrum intersects the straight tab attached to the apex of the triangle and the resolution improves to $\Delta B=0.44$ and $0.40 \mathrm{~nm}$, respectively. In each graph in Fig. 4, the dashed curve shows an appropriate filter setting for demultiplexing $50-\mathrm{GHz}$ spaced channels. A filter with a bandwidth adjustable in discrete steps would show resolution approaching the theoretical limit.

Bit-error-rate (BER) testing at $10 \mathrm{~Gb} / \mathrm{s}$ verified the suitability of this filter for high bandwidth data transmission. The power penalty was measured as a function of detuning from the center of the channel passband (Fig. 5). The penalty is expected to be low due to the inherently low group-delay ripple of the design, and, in fact, the penalty for $10^{-9}$ BER is under $0.1 \mathrm{~dB}$ for channel detuning across the filter's $23.5-\mathrm{GHz} 1-\mathrm{dB}$ bandwidth. The adjacent channel isolation is $>30 \mathrm{~dB}$ for $50-\mathrm{GHz}$ spaced channels.

The architecture presented in Fig. 1 can be extended to a three-port "hitless" tunable drop filter by adding an "expressport" fiber. Wavelength signals $\lambda_{1}$ and $\lambda_{3}$ reflected by the SPSF outer field region would now be directed to this fiber rather than discarded. Tilting the mirror about the $y$-axis so that the imaged spectrum moves off the end of the tilted SPSF beam allows wavelength tuning without express-channel interruption. Large incident-angle-dependence of the diffraction efficiency renders the current grating unsuitable for this application. However, we demonstrate the principle of an SPSF-based drop switch using the setup of Fig. 6(a). The configuration is the same as that in Fig. 1 except that a third express-port fiber is added and the SPSF is moved to the input image plane, eliminating the grating and MEMS. The anamorphic condensing lens focuses light from the input fiber onto the SPSF. The tilted beam (rectangular in this case) reflects light to the "drop" port while the surrounding field reflects light to the "express" port. To ensure low crosstalk, the drop channel signal must be well-centered on the beam. A photodiode (PD) placed behind the SPSF measures light leaking through the $\sim 2-\mu \mathrm{m}$ gap at the perimeter of the beam. The power measured by this diode correlates with the crosstalk into the express port as the SPSF is translated laterally in the $y$-direction [Fig 6(b)]. Stray light limits the PD sensitivity. In an actual drop filter, complete with grating and MEMS mirror, the photocurrent could feedback-stabilize the mirror, locking onto the drop channel.

\section{CONCLUSION}

We have demonstrated a two-port spectral filter that uses a single two-axis MEMS tilt mirror to continuously tune center wavelength across $36 \mathrm{~nm}$, and independently tune passband $3-\mathrm{dB}$ spectral width from 28 to $210 \mathrm{GHz}$. The $10-\mathrm{Gb} / \mathrm{s}$ power-penalty is under $0.1 \mathrm{~dB}$ across the passband. The worst-case IL and PDL (4.6 and $0.26 \mathrm{~dB}$ ) were dominated by the diffraction grating.

This result is the first demonstration of a new class of compact multifunctional filters that includes three-port channel drop filters as well as two-port bandpass filters, using a single tilt mirror in a double-pass spectrometer to select among preprogrammed spectral filters. Spectrum steering devices may utilize active feedback to maintain alignment of the system over extremes in temperature, eliminating the need for an expensive package sufficiently athermal to maintain micrometer scale alignments over long optical path lengths.

\section{REFERENCES}

[1] J. Berger, F. Ilkov, D. King, A. Tselikov, and D. Anthon, "Widely tunable, narrow optical bandpass Gaussian filter using a silicon microactuator," in Proc. OFC, 2003, pp. 252-253.

[2] K. Nakamura, T. Saitoh, and Y. Takahashi, "High-speed optical performance monitor for WDM network using MEMS scanning mirror," in Proc. IEEE/LEOS Int. Conf. Optical MEMS, 2003, pp. 97-98.

[3] F. Zimmer, H. Grüger, A. Heberer, A. Wolter, and H. Schenk, "Development of a NIR micro spectrometer based on a MOEMS scanning grating," in Proc. SPIE Conf. MEMS, MOEMS, and Micromachining, 2004, pp. 6114-6119.

[4] J. E. Ford, V. A. Aksyuk, D. J. Bishop, and J. A. Walker, "Wavelength add-drop switching using tilting micromirrors," J. Lightw. Technol., vol. 17, no. 5, pp. 1663-1670, May 1999.

[5] J. Kondis, B. A. Scott, A. Ranalli, and R. Lindquist, "Liquid crystals in bulk optics-base DWDM optical switches and spectral equalizers," in Proc. 14th Annu. Meeting IEEE Lasers and Electro-Optics Soc., 2001, vol. 1, pp. 292-293.

[6] A. Boskovic, M. Sharma, N. Antoniades, and M. Lee, "Broadcast and select OADM nodes: Application and performance trade-offs," in Proc. OFC, 2002, pp. 159-159.

[7] R. Ryf, Y. Su, L. Möller, S. Chandrasekhar, D. T. Neilson, and C. R. Giles, "Data rate and channel spacing flexible wavelength blocking filter," in Proc. OFC, Los Angeles, CA, 2004, Paper PDP10.

[8] D. M. Marom, D. T. Neilson, D. S. Greywall, V. A. Aksyuk, M. E. Simon, N. R. Basavanhally, P. R. Kolodner, Y. L. Low, F. Pardo, C. A. Bolle, C. S. Pai, D. López, J. A. Taylor, E. J. Bower, J. Leuthold, M. A. Gibbons, and C. R. Giles, "Wavelength selective $4 \times 1$ switch with high spectral efficiency, $10 \mathrm{~dB}$ dynamic equalization range and internal blocking capability," in Proc. ECOC, Rimini, Italy, 2003, Paper Mo3.5.3. 